\title{
Challenges of Hand Hygiene in Healthcare: The Development of a Tool Kit to Create Supportive Processes and Environments
}

Anjum Chagpar, Carleene Banez, Raquel Lopez and Joseph A. Cafazzo

\section{Abstract}

Hand hygiene compliance by healthcare providers has been difficult to achieve due to diverse environments, work culture, processes and task requirements. Because of this complexity, hand hygiene lends itself well to a human factors analysis in order to design a system that matches human cognitive and physical strengths and makes allowances for human limitations.

\section{A} multi-phased user-centred approach was undertaken to explore barriers and enablers to hand hygiene in diverse environments (rehabilitation, family medicine, emergency and intensive care) for a number of healthcare workers (HCWs; physicians, nurses, allied health, housekeeping and patient support workers). Observational studies, interviews, focus groups and surveys were used to engage end users in solution development. Solutions were then validated through an environmental modification study, which sought to quantify the benefits of proposed solutions.

This research highlighted the need to take into consideration the differences between HCWs, their environments and the tools with which they are provided when recommending solutions to mitigate barriers. Context-specific recommendations resulting from this work have been formulated into a tool kit for dissemination by the Canadian Patient Safety Institute (CPSI).

\section{Background}

CPSI has partnered with provincial governments to encourage HCWs to adhere to the "four moments of hand hygiene":

- Before initial contact with a patient or patient's environment

- Before performing an aseptic procedure

- After the risk of body fluid exposure

- After contact with a patient or patient's environment

Compliance is a challenge, however. While it is well known that proper hand hygiene practices are the most effective method of reducing hospital-acquired infections (HAIs), the rate with which HCWs comply with best practice recommendations is still only approximately $40 \%$ (World Health Organization 2005). Low compliance is one reason that $5-10 \%$ of patients admitted into hospitals acquire at least one HAI (World Health Organization 2005).

There is no shortage of initiatives to address low compliance (Table 1). Best practice guidelines, education campaigns and guidance on auditing compliance are widespread. Yet existing recommendations around best practices often conflict, creating confusion for HCWs. For example, plain soap has been recommended because it is less likely to cause dermatitis (Jumaa 2005). Yet handwashing with plain soap does not remove pathogens, a fact that has resulted in recommendations to use antimicrobial 
soaps and alcohol-based hand rubs (ABHRs). Similarly, because sink faucets can contaminate clean hands, guidelines recommend that sinks be sensor operated (Cochrane 2003). This is in conflict with reports that electronic faucets are more likely to harbour bacteria, which has resulted in a recommendation that sinks with manual faucets be employed (Merrer et al. 2005).

While there is support for the development of gold standards for hand hygiene (Elliott 2003; Farrington 2007; Larson 2003; Macias and Ponce-De-Leon 2005; Seal et al. 2005), some believe that it is necessary for providers to develop their own institution-appropriate guidelines (Held et al. 2001) and that $100 \%$ compliance may interfere with patient care (Storr and Clayton-Kent 2004).

Instead of focusing on education or auditing campaigns, or further developing and clarifying best practice guidelines, this project sought to identify barriers and enablers of hand hygiene in order to make environments and processes more supportive of hand hygiene activities. This human factors approach aims to optimize environments and processes that are natural and easy to use by matching them to human cognitive and physical strengths and making allowances for human limitations.

\section{Methods}

Five methods were used to identify barriers and enablers to performing hand hygiene, to design and validate potential solutions and to create a tool kit for healthcare institutions based on the project learnings:

1. A literature review to understand the current state with respect to barriers and enablers to hand hygiene

2. Heuristic evaluations of common hand hygiene products to identify features that positively or negatively influence performance and compliance

3. Field studies to determine the workflow of various HCWs so that barriers and enablers could be contextualized

4. Focus groups with HCWs to brainstorm and validate the potential of proposed solutions to the barriers identified using the previous three methods

5. An environment modification and validation study in which patterns of usage were monitored and qualitative findings from HCWs were sought through surveys

Table 1. Best practice guidelines and campaigns

\begin{tabular}{|c|c|c|}
\hline Organization or Country & Year Published & Title of Article \\
\hline World Health Organization & 2005 & WHO Guidelines on Hand Hygiene in Health Care \\
\hline $\begin{array}{l}\text { Centers for Disease Control and Prevention - United } \\
\text { States }\end{array}$ & 2003 & $\begin{array}{l}\text { Guideline for Hand Hygiene in Health-Care Settings: Recommendations } \\
\text { of the Healthcare Infection Control Practices Advisory Committee and } \\
\text { the HICPAC/SHEA/APIC/IDSA }\end{array}$ \\
\hline Ontario Ministry of Health and Long-Term Care & 2008 & Best Practices for Hand Hygiene In All Health Care Settings \\
\hline Ireland & 2001 & Guidelines for Hand Hygiene in Irish Health Care Settings \\
\hline $\begin{array}{l}\text { National Institute for Clinical Excellence: United } \\
\text { Kingdom }\end{array}$ & 2003 & $\begin{array}{l}\text { Infection Control: Prevention of Healthcare-Associated Infection in } \\
\text { Primary and Community Care }\end{array}$ \\
\hline Australia & 2004 & Infection Control \\
\hline $\begin{array}{l}\text { Centers for Disease Control, Association for } \\
\text { Professionals in Infection Control and Epidemiology, } \\
\text { and Society for Healthcare Epidemiology of America }\end{array}$ & 2006 & How-to Guide: Improving Hand Hygiene \\
\hline Public Health Agency of Canada & 1998 & Hand Washing, Cleaning, Disinfection and Sterilization in Health Care \\
\hline Department of Health - England & 2003 & $\begin{array}{l}\text { Winning Ways: Working together to reduce Healthcare Associated } \\
\text { Infection in England }\end{array}$ \\
\hline American Institute of Architects & 2006 & $\begin{array}{l}2006 \text { Guidelines for Design and Construction of Hospital and Healthcare } \\
\text { Facilities }\end{array}$ \\
\hline National Health Service - United Kingdom & 2008 & Infection Control in the Built Environment: Design and Briefing \\
\hline
\end{tabular}




\section{Literature Review}

A literature search was performed using Medline, CINAHL and Embase databases. Separate searches were conducted for hand washing, hand disinfection, attitudes, devices and human factors.

Two additional restrictions were placed to help focus the search: only English articles and articles published since January 2000 were included. Using Medline, CINAHL and Embase, a total of 292, 163 and 306 articles were found, respectively. The titles and abstracts of the results from the three databases were examined. Bibliographical information from relevant articles was noted, and duplicates were eliminated. Finally, articles were excluded if they (1) were conducted in dental surgeries or (2) centred on surgical scrubbing and hand preparation. The remaining number of noted articles requiring further analysis was 111 .

\section{Heuristic Evaluation}

Heuristic evaluation refers to the systematic inspection of a user-interface design for usability. Using a checklist of usability principles (or heuristics) as a guide, a product is evaluated according to how well it satisfies each principle. Common heuristics include accessibility, visibility, consistency, autonomy, efficiency, flexibility and error prevention (Zhang et al. 2003).

A heuristic evaluation was conducted to assess the hand hygiene products used in four clinical areas: the Medical Surgical Intensive Care Unit (MSICU), the Emergency Department (ED) and the Family Medicine Clinic at University Health Network, and the Medical Activation and Rehabilitation Unit at Bridgepoint Health Centre, in Toronto, Ontario. Between these four units, three types of sinks, four types of waste receptacles, four ABHRs, three brands of gloves, one lotion and two soap products were evaluated. The evaluations were conducted independently by two human factors specialists, who then came to consensus on identified issues through subsequent deliberation.

\section{Field Studies}

Field studies were conducted in the same four clinical units that were reviewed during the heuristic evaluations. A total of 110 hours of direct observation of members of the nursing, physician, allied health, patient support worker and housekeeping populations were conducted. Two human factors specialists concurrently observed HCWs so that significant findings could be discussed and consensus on barriers and enablers reached. Following the shadowing sessions, in-context interviews were used to further explore observations and understand decisionmaking rationale and context. Workflow maps were then developed to understand where hand hygiene should occur and to discover trends and root causes for poor compliance.

\section{Focus Groups}

A total of six focus groups were conducted with allied health professionals, rehabilitation HCWs, family medicine HCWs, housekeeping staff, ED and MSICU nurses and ED and MSICU physicians. Where possible, groups consisted of only one profession in order to avoid potential inter-group effects and to encourage open dialogue around profession-specific barriers and enablers. Each session involved between six and eight participants who were asked to validate observed barriers and enablers and provide feedback on their generalizability and comprehensiveness. In addition, these sessions were used to brainstorm solutions that would then be selected for inclusion in the environmental modification and validation study.

\section{Environment Modification and Validation Study}

Following the design of potential solutions, an environment modification study was undertaken. In each of the four areas of study, two rooms were modified based on the results and recommendations of the literature review, heuristic evaluations, field studies and focus groups. These changes differed depending on the unit owing to the nature of their current physical environment. In all units, additional ABHR dispensers or bottles with redesigned labels were installed and additional products and accessories were relocated (regarding height, surface mounts etc.). Over a period of 18 days, data from digital dispensing counters were collected on the amount of product used. After the study period, these data were employed to determine which ABHRs were used most frequently. Post-modification surveys were also administered to all staff to assess their perceptions of the changes.

\section{Findings \\ Literature Review}

HCWs rightly view hand hygiene as a means of preventing the spread of infections to patients (Creedon 2006; O'Boyle et al. 2001a ). In addition, they regard hand hygiene as a method of protecting themselves and their colleagues from acquiring infections (Creedon 2006; Lankford et al. 2003; O'Boyle et al. 2001a; Whitby et al. 2007). Still, compliance has been difficult to achieve.

O'Boyle et al. (2001b) used the Theory of Planned Behaviour to develop a model of the internal factors that motivate hand hygiene, including belief in the effectiveness of hand hygiene in reducing HAIs, the perception of social pressure to perform hand hygiene and the perceived ease of adding hand hygiene into workflow. These three factors influence the intention to perform hand hygiene. A fourth factor is the intensity of activity.

Extending O'Boyle et al.'s (2001b) model to infer external factors that influence hand hygiene performance, we hypothesized that factors such as the design of environments and processes could have an influence of the perceived ease of adding hand hygiene into the workflow as well as on the intensity of activity (Figure 1).

Suresh and Cahill (2007) and Cochrane (2003) used 
Figure 1. Modified Theory of Planned Behaviour

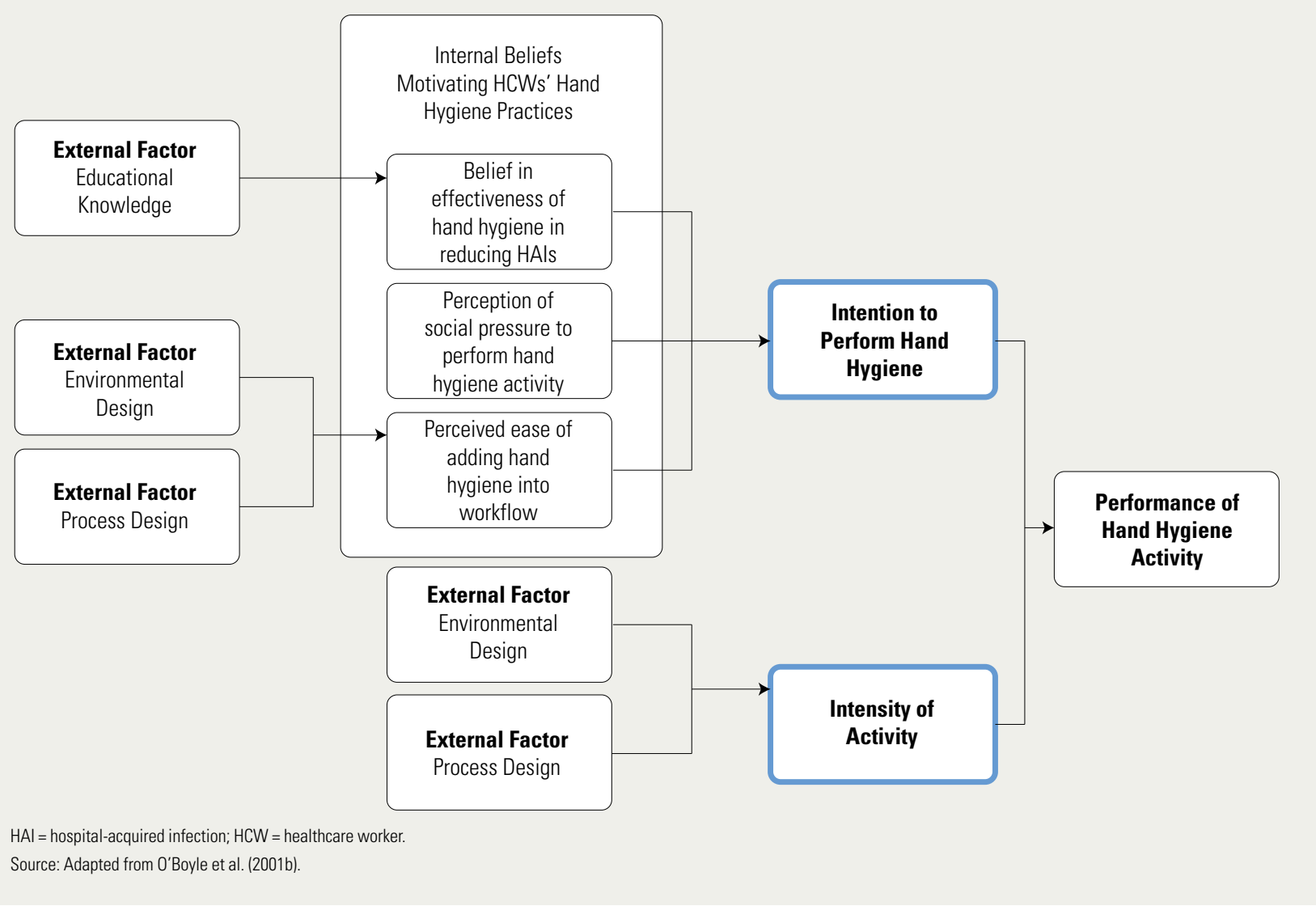

human factors principles to investigate four of the environmental barriers: sinks, waste receptacles, ABHRs and gloves. No other studies were found that used human factors principles to assess and improve environments and processes related to hand hygiene.

\section{Field Studies}

Findings from direct observation sessions were in alignment with research reporting that healthcare professionals work in environments that do not support high-quality hand hygiene practices (Suresh and Cahill 2007). Thematic analysis of observational notes and in-context interview transcripts revealed three classes of barriers: environmental, attitudinal and process. Examples of each are described below.

\section{Environmental Barriers}

When exiting isolation rooms, HCWs remove personal protective equipment such as gowns, gloves and masks at the doorway. In accordance with recommended guidelines, once their equipment is removed, they are expected to perform hand hygiene.
This is difficult to do in many units as sinks are frequently located at the back of patients' rooms (Figure 2). Staff admitted that they re-enter the room to wash their hands or search for a sink in another location as a workaround. However, in their busy work environments, HCWs were often distracted on their way to find a sink and, as a result, started a new task without performing hand hygiene.

\section{Attitudinal Barriers or Beliefs}

In addition to environmental barriers, shadowing revealed several attitudinal barriers or beliefs about hand hygiene that prevented compliance with best practices. For example, it was observed that staff members were usually compliant with performing hand hygiene after glove removal. However, their compliance before donning gloves was much lower. Follow-up in-context interviews revealed that it is very difficult to don gloves when hands are damp, and HCWs rarely have enough time to wait for their hands to dry completely. Many perceived it to be more important to perform their patient care task quickly while wearing gloves than to perform hand hygiene 
Figure 2. Poor access to sink located at the back of room, making it difficult to perform hand hygiene upon entering and exiting patient environment

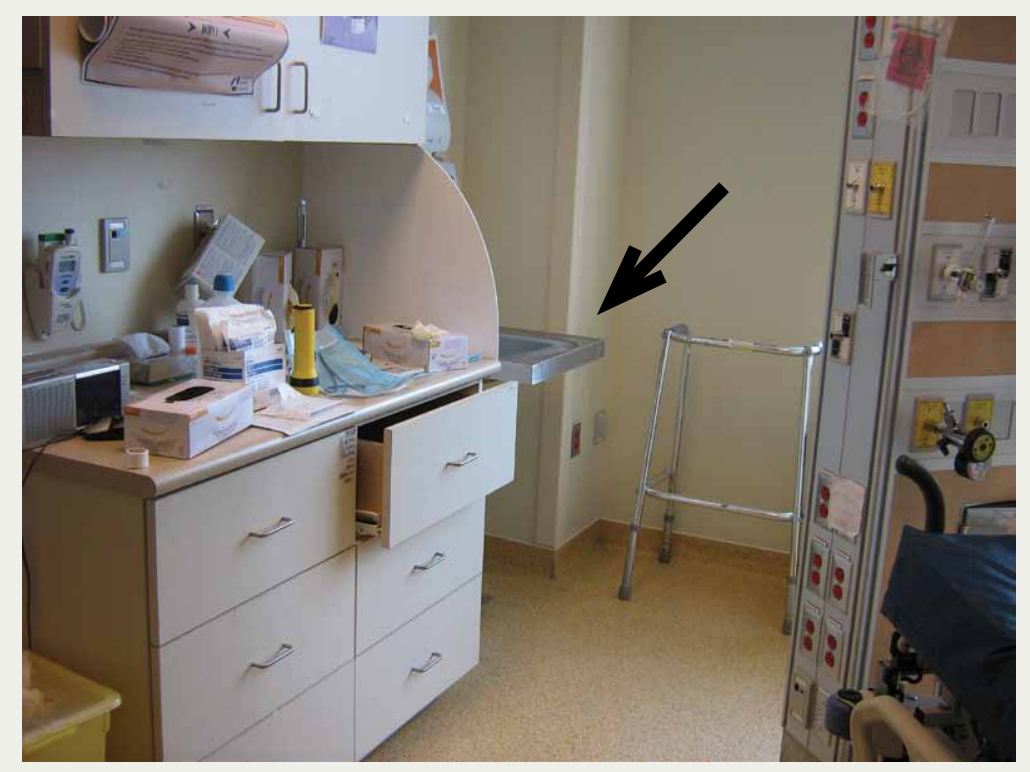

before donning gloves to mitigate the risk of infection through small defects in the gloves.

\section{Process Barriers}

A third type of barrier identified was a process barrier - a routine put in place by a healthcare institution that discourages hand hygiene practices. For example, while in a patient's room, an HCW may take vital signs, check urine output and rotate the patient. According to the four moments of hand hygiene, hand cleansing should occur before entering the patient's environment, after the risk of exposure to body fluid and after exiting the patient's environment (Ontario Ministry of Health and Long-Term Care 2008). A policy requiring that gloves be worn made it tedious to perform hand hygiene for all the recommended moments as the gloves had to be removed and placed in a waste bin, hand hygiene products had to be located and used and new gloves needed to be donned before care could be continued. Many staff members were unable to comply with

Figure 3. Look-alike, unlabelled soap and ABHR dispensers

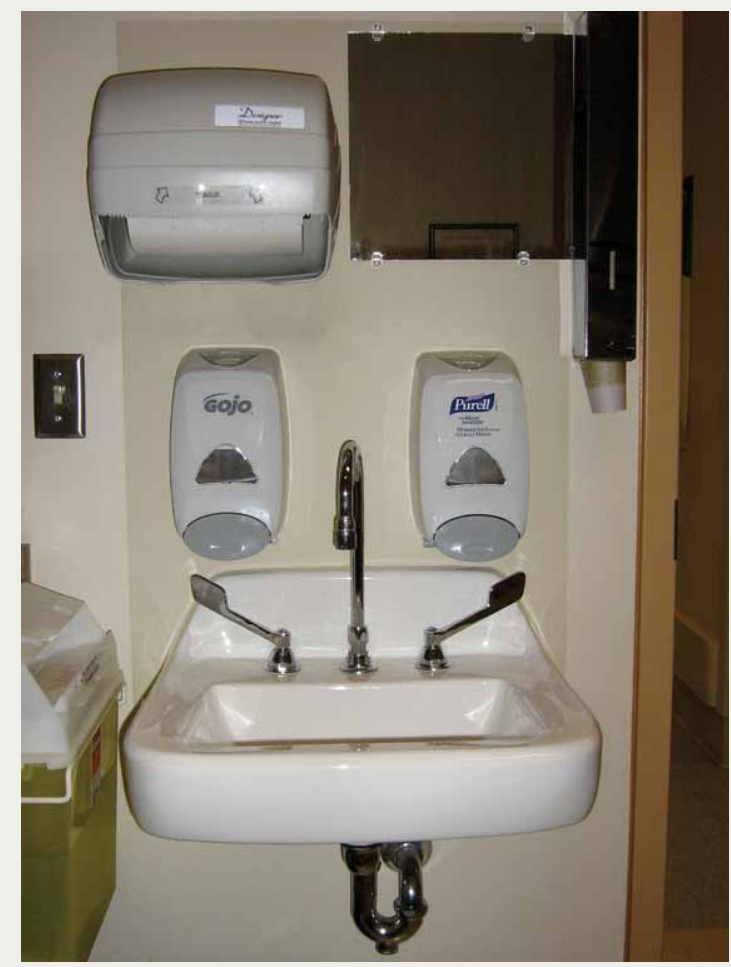

$\mathrm{ABHR}=$ alcohol-based hand rub the recommended guidelines because of the additional time it required to complete this process.

The three types of barriers identified were very much related. Environmental barriers often reinforce attitudinal and process barriers. Not having the appropriate hand hygiene products always available forces HCWs to use products that are inferior as a substitute or to omit hand-cleaning practices when they believe it is not essential (Lankford et al. 2003; Suresh and Cahill 2007). When products are placed in inconvenient locations, this increases the length of time it takes to perform the task (Cochrane 2003).

\section{Heuristic Evaluation}

Several significant findings that influenced the ease with which hand hygiene was performed were found. With respect to soap, ABHRs and lotions, all three types of dispensers were the same size, shape and colour and had the same actuation method (Figure 3). This made it easy for products to be mistaken for each other and thus be used inappropriately.

Products were to be identified by brand names (Purell and GoJo) instead of by product. Small windows made it difficult to determine the amount of product remaining.

Another significant problem with the dispensers was the inability to view the remaining liquid levels (see Figure 3). Although the dispensers had clear plastic windows, it was difficult 
to see the transparent liquid through the small opening. In order to view the amount of product remaining, the dispenser had to be fully opened, which increased the time of the refilling process as well as the likelihood of cross-contamination when empty containers were actuated.

Examples of other issues found include the inconsistent mounting height of the alcohol-based hand sanitizers, sometimes as high as $145 \mathrm{~cm}$ (57 inches), and the lack of temperature and pressure control on hand-free sinks. In order to be accessible for the average adult population, dispensers should be mounted at a height of between 84 and $112 \mathrm{~cm}$ (33-44 inches). Hands-free sinks may discourage handwashing if the water is either too hot or too cold, or if the water pressure is too high or too low.

\section{Focus Groups}

Several focus groups were held with each of the user groups to validate findings, share learnings and brainstorm solutions. These member-checking sessions revealed insights into how potential barriers may be addressed. For example, during observational studies, users were seen to be spending more time on handwashing in bathrooms while observing their reflection in a mirror. Sharing this finding during the brainstorming sessions led to the idea to place mirrors above ABHRs. Focus groups with housekeeping staff resulted in the development of a pop-up "EMPTY" flag to be used by HCWs when they attempt to dispense a product from an empty container (Figure 4). This approach is meant to engage everyone in the environment in the maintenance of full dispensers by also providing a number to call for a refill.
Mirrors were added above dispensers of alcohol-based hand rub to incent use. "EMPTY" flags were added to the dispensers to involve all users in keeping them full.

Focus groups were also used to validate and refine the design of new labels for soap, ABHRs and lotions in order to make them easier to identify. While best practice graphic design and human factors guidelines (Smith 1979; Wiednbeck 1999; Woodson and Conover 1964) were applied to the design, the focus groups yielded important insights into the most intuitive colours for the labels: pink for soap, yellow for lotion and blue for ABHRs.

\section{Environmental Modification and Validation Study}

In all units modified, additional ABHR dispensers or bottles with redesigned labels were installed, and additional products and accessories were relocated (regarding heights, surface mounts etc.). Digital actuation counters were installed on these ABHRs so that the number of times they were used during the study period could be captured. These frequency data were then used to identify optimal locations (Figure 5).

The locations and numbers of additional products were not meant to be ideal. Instead, they were used to explore behaviour patterns in order to identify locations and products that were used most frequently.

Data from the ABHR actuation counters revealed that the ABHRs placed just outside the room were used the most frequently. There was less agreement on where the optimal locations were for products inside the rooms. Surveys indicated that, even within a particular unit, physicians and nurses disagreed as to their preferred location of products, likely due to their differing workflows. Different preferences were also seen across the various locations, although there was universal consensus that ABHRs be placed at specimen drop-off, pneumatic tube and blood analysis machine locations.

The product empty flags were not felt to be visible enough, and observations confirmed that these were rarely used. Mirrors placed above the ABHRs were also not found to affect the frequency of use. Qualitative survey data revealed this to be due to the lack of privacy with which to view oneself in a hallway or patient room as compared with a washroom.

To address the variability in requirements of locations as well as the shortcomings of some of the potential solutions, recommenda- 
tions were iteratively developed with HCWs from each of the environments. For example, we learned of a method to engage HCWs in the optimal placement of products using stickers. Each HCW was given a set of coloured dot stickers, each colour representing a different hand hygiene product. For a period of two weeks, whenever a hand hygiene product was unavailable at a particular location, HCWs were asked to place a dot at their preferred location. After two weeks, the densest clusters of dots were identified and used to determine product placement. Data collected after the modifications revealed a high degree of satisfaction with the re-worked environment.

\section{Discussion}

This study found that some recommendations for hand hygiene environment and process optimization were universal:

- At least one ABHR should be located within arm's reach of a patient room door.

- Dispensers for different products should be distinctly different to avoid product confusion.

- Glove box containers should be mounted on vertical surfaces to increase accessibility and visibility.

It also highlighted that many recommendations are highly context dependent. To address this issue, a tool kit was developed to enable HCWs to apply universal recommendations as well as develop their own optimizations through the application of human factors principles. The development of the tool kit involved input from diverse stakeholders to ensure that its recommendations were sound and consistent with best practice guidelines. Ten infection prevention and control departments throughout Canada were sent content drafts and asked to provide feedback through a survey.

The resulting tool kit contains three sub-tools. The Environment Assessment Tool provides guidelines for creating environments that optimally support hand hygiene activities. Each recommendation in the guideline includes a human factors rationale and cites the usability principles (e.g., visibility, consistency, efficiency, flexibility etc.) that it meets. This context is provided to allow HCWs to adapt the recommendation if necessary, while maintaining the human factors and usability benefits. It is also intended to help alleviate confusion when guidelines may appear to conflict. For example, it may be sufficient to use manual sinks with long-lever faucets if they are in close proximity to paper towels and waste receptacles are appropriately located. Alternatively, if electronic sinks are used, they should have temperature and flow controls that are easily accessible, visible and understandable so that they can be changed according to individual preferences.

The Environment Assessment Tool also includes recommendations on how to engage front-line HCWs in the identification of ideal locations for products in their environments. Similarly, the Product Selection Tool provides guidance on how to engage HCWs in product procurement as well as label design, and the Maintenance Process Tool includes guidelines to collaboratively develop processes for ensuring the supply and maintenance of hand hygiene products.

The tools provided in the tool kit are consistent with the usercentred approach applied in this project to develop solutions. They aim to involve end users in the application of the recommendations according to their specific contexts as well as in the development of new solutions through focus groups and other collaborative activities. These approaches, along with the human factors and usability principles, applied to the optimiza-

\section{Figure 5. A quad-bed rehabilitation room before and after modifications}
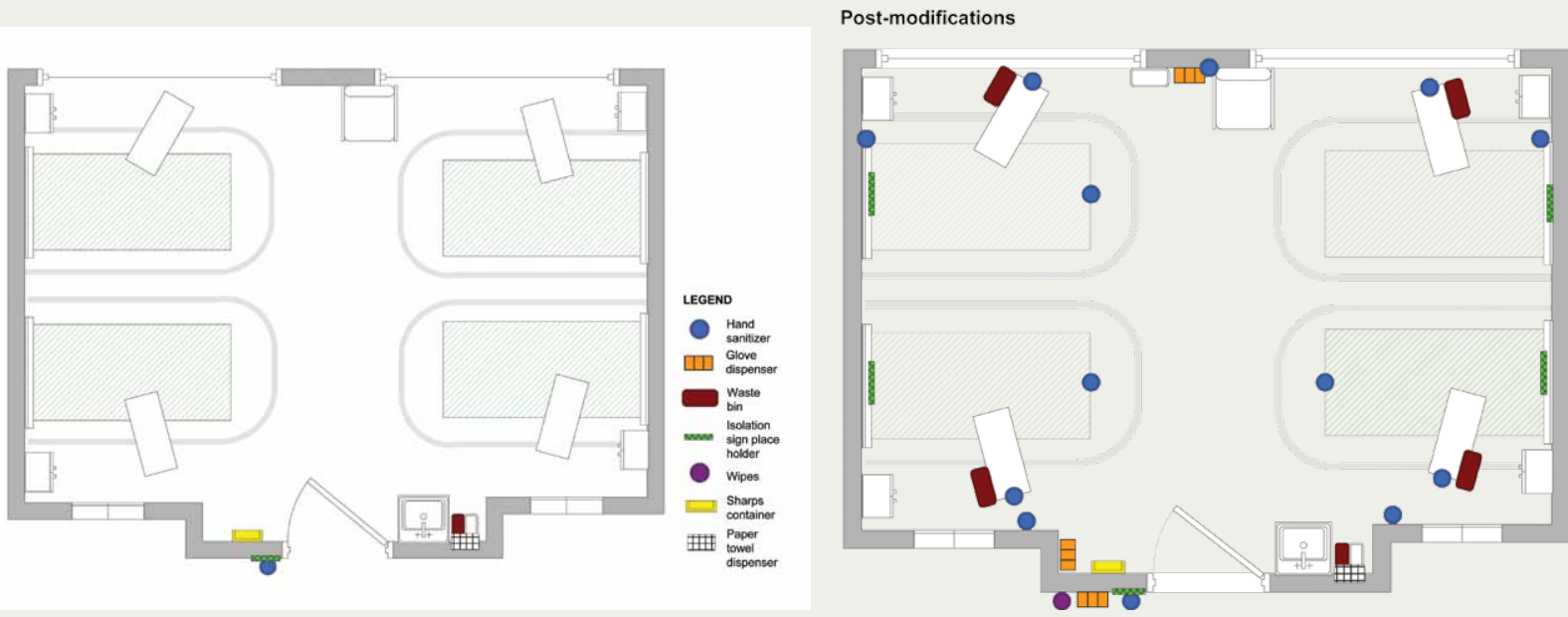
tion of hand hygiene environments and processes may also be applied to other clinical processes and environments. By understanding the particular limitations and needs of end users and developing solutions collaboratively with them, human factors approaches result in user-validated customized solutions that better meet the needs of HCWs.

While the focus of this tool kit is on external factors that contribute to hand hygiene performance (see Figure 1), future work on further understanding and addressing the internal factors that influence hand hygiene is needed to fully address the issue of low compliance.

The tool kit was launched in April 2010 at Canada's Forum on Patient Safety and Quality Improvement. Copies are available from http://www.saferhealthcarenow.ca/EN/HandHygiene/ Pages/HumanFactorsToolKit.aspx. HQ

\section{Acknowledgements}

This study was funded in part by the Canadian Patient Safety Institute. The authors would also like to acknowledge the work of Stephanie Liddle in data collection and analysis, as well as the study participants. Finally, the authors are grateful for the support of the Ontario College of Art's Industrial Design Department's faculty and students.

\section{References}

Cochrane, J. 2003. "Infection Control Audit of Hand Hygiene Facilities." Nursing Standard (Royal College of Nursing [Great Britain]) 17(18): 33-38.

Creedon, S.A. 2006. "Infection Control: Behavioural Issues for Healthcare Workers." Clinical Governance 11(4): 316-25.

Elliott, P. 2003. "Recognising the Psychosocial Issues Involved in Hand Hygiene.” Journal of the Royal Society of Health 123(2): 88-94.

Farrington, M. 2007. "Infection Control Education: How to Make an Impact - Tools for the Job.” Journal of Hospital Infection 65(Suppl 2): $128-32$.

Held, E, C. Wolff, F. Gyntelberg and T. Agner 2001. "Prevention of Work-Related Skin Problems in Student Auxiliary Nurses: An Intervention Study." Contact Dermatitis 44(5): 297-303.

Jumaa, P. A. 2005. Hand Hygiene: Simple and Complex. International Journal of Infectious Diseases: 9(1), 3-14.

Lankford, M.G., T.R. Zembower, W.E. Trick, D.M. Hacek, G.A. Noskin and L.R. Peterson. 2003. "Influence of Role Models and Hospital Design on Hand Hygiene of Healthcare Workers." Emerging Infectious Diseases 9(2): 217-23.

Larson, E. 2003. "Status of Practice Guidelines in the United States: CDC Guidelines as an Example.” Preventive Medicine 36(5): 519-24.

Macias, A.E. and S. Ponce-De-Leon. 2005. "Infection Control: Old Problems and New Challenges." Archives of Medical Research 36(6): 637-45.

Merrer, J., E. Girou, D. Ducellier, N. Clavreul, F. Cizeau, P. Legrand et al. 2005. "Should Electronic Faucets Be Used in Intensive Care and Hematology Units?.” Intensive Care Medicine 31(12): 1715-18.

O’Boyle, C.A., S. Henly and L.J. Duckett. 2001a. "Nurses' Motivation to Wash Their Hands: A Standardized Measurement Approach."
Applied Nursing Research: ANR 14(3): 136-45.

O’Boyle, C.A., S.J. Henly and E. Larson. 2001b. "Understanding Adherence to Hand Hygiene Recommendations: The Theory of Planned Behavior." American Journal of Infection Control 29(6): 352-60.

Ontario Ministry of Health and Long-Term Care. 2008. Best Practices for Hand Hygiene: In All Health Care Settings. Toronto, ON: Provincial Infectious Diseases Advisory Committee.

Seal, L.A., R.L. Rizer and R. Maas-Irslinger. 2005. "A Unique Water Optional Health Care Personnel Handwash Provides Antimicrobial Persistence and Residual Effects While Decreasing the Need for Additional Products." American Journal of Infection Control 33(4): 207-16.

Smith, S.L. 1979. "Letter Size and Legibility.” Human Factors 21(6): 661-70.

Storr, J. and S. Clayton-Kent. 2004. "Hand Hygiene." Nursing Standard 18(40): 45-52

Suresh, G. and J. Cahill. 2007. "How 'User Friendly' Is the Hospital for Practicing Hand Hygiene? An Ergonomic Evaluation.” Joint Commission Journal on Quality and Patient Safety 33(3): 171-79.

Whitby, M., C.L. Pessoa-Silva, M.L. McLaws, B. Allegranzi, H. Sax, E. Larson et al. 2007. "Behavioural Considerations for Hand Hygiene Practices: The Basic Building Blocks." Journal of Hospital Infection 65(1): 1-8.

Wiedenbeck, S. 1999. "The Use of Icons and Labels in an End User Application Program: An Empirical Study of Learning and Retention." Behaviour and Information Technology 18(2): 68-82.

Woodson, W.E. and D.W. Conover. 1964. Human Factors Engineering Guide for Equipment Designers (2nd ed.). Berkeley, CA: University of California Press.

World Health Organization. 2005. World Alliance for Patient Safety. Global Patient Safety Challenge 2005-2006: Clean Care Is Safer Care. Geneva, Switzerland: WHO Document Production Services.

Zhang, J., T.R. Johnson, V.L. Patel, D.L. Paige and T. Kubose. 2003.

"Using Usability Heuristics to Evaluate Patient Safety of Medical Devices." Journal of Biomedical Informatics 36(1-2): 23-30.

\section{About the Authors \\ Anjum Chagpar, MHSc, PEng, is a member of Healthcare Human Factors, University Health Network, in Toronto, Ontario.}

Carleene Banez, BEng, is a member of Healthcare Human Factors, University Health Network.

Raquel Lopez, MHSc, is a former member of Healthcare Human Factors, University Health Network, and is currently pursuing a degree in medicine.

Joseph A. Cafazzo, PhD, PEng, is a member of Healthcare Human Factors, University Health Network; the Department of Health Policy, Management and Evaluation, Faculty of Medicine, University of Toronto; and the Institute of Biomaterials and Biomedical Engineering, Faculty of Medicine, University of Toronto. 